\title{
Position measurement of a dipolar scatterer via self-homodyne detection
}

\author{
G. Cerchiari $\odot,{ }^{1,{ }^{*}}$ L. Dania $\odot,{ }^{1}$ D. S. Bykov $\odot,{ }^{1}$ R. Blatt,${ }^{1,2}$ and T. E. Northup $\odot^{1}$ \\ ${ }^{1}$ Institut für Experimentalphysik, Universität Innsbruck, Technikerstrasse 25, 6020 Innsbruck, Austria \\ ${ }^{2}$ Institut für Quantenoptik und Quanteninformation, Österreichische Akademie der Wissenschaften, \\ Technikerstrasse 21a, 6020 Innsbruck, Austria
}

(Received 16 March 2021; revised 28 September 2021; accepted 20 October 2021; published 19 November 2021)

\begin{abstract}
We describe a technique to measure the position of a dipolar scatterer based on self-homodyne detection of the scattered light. The method can theoretically reach the Heisenberg limit, at which information gained about the position is constrained only by the back action of the scattered light. The technique has applications in the fields of levitated optomechanics and trapped ions and is generally applicable to the position determination of confined light scatterers.
\end{abstract}

DOI: 10.1103/PhysRevA.104.053523

\section{INTRODUCTION}

One of the new frontiers in physics is to explore the boundaries between quantum and classical theories and to understand the role of gravity in the quantum-mechanical description [1,2]. A promising approach in these investigations is to search for quantum-mechanical properties of the motion of micron- or submicron-sized dielectric particles levitated in optical, magnetic, or electrodynamic traps $[3,4]$. The levitation ensures a high degree of isolation in space and enables the study of the particles' wave properties stemming from the quantization of the confining potential [5-7]. In these systems, laser light is typically used to measure and interact with the levitated particle's motion. The standard optical approach to observe single quanta of oscillatory motion (phonons) is to detect a monochromatic light field scattered by the particle [8-10]. The scattered field carries information about the location of emission but, at the same time, perturbs the momentum of the particle via photon recoil [11]. The trade-off between the information gained and the back action sets the ultimate limit for detection: the Heisenberg limit. Here, by Heisenberg limit, we mean that the product of the measurement imprecision in the determination of the position and the measurement back action on the scatterer's momentum satisfies the lower bound of the Heisenberg uncertainty relation [12]. A further challenge is imposed by the angular distribution of the scattered light, which, in the Rayleigh approximation, is a dipole pattern that spreads the information over the full solid angle [13].

Observation of the scattered light over the entire solid angle is the key to reaching the Heisenberg limit as proposed in Ref. [14]. In the gedanken experiment described

\footnotetext{
*Corresponding author: giovanni.cerchiari@uibk.ac.at
}

Published by the American Physical Society under the terms of the Creative Commons Attribution 4.0 International license. Further distribution of this work must maintain attribution to the author(s) and the published article's title, journal citation, and DOI. therein, the following geometrical configuration is proposed. The scattered field is interfered with a second dipolar field having the same wave fronts and polarization, allowing for the reconstruction of the scatterer's position via homodyne detection. The secondary dipole field (reference field) must be permanently located at a reference position, which defines the origin of the scatterer's displacements. This theoretical configuration poses the challenge of generating the dipolar reference field while simultaneously detecting light over the entire solid angle. This optical problem has been approached in the past by locating the scatterers in the focus of deep parabolic mirrors to collimate the dipolar field into a plane wave having a Gaussian $\mathrm{TEM}_{01 *}$ profile, a so-called doughnut mode $[15,16]$. This solution requires the parabolic mirror to cover a large fraction of the solid angle around the scatterer to collimate the field and may be difficult to implement in experiments with limited optical access.

In this work, we follow a different approach based on spherical optics and analyze its theoretical limitations for position and motion detection of a dipolar scatterer. The solid angle is split into two parts. One part is occupied by a mirror, the other by a detector. The mirror generates a secondary image of the dipole, which interferes at the detector with the primary scattered light. Such an optical configuration realizes the self-homodyne of the scattered field and it has been explored in the past to obtain nanometric resolution in microscopy applications $[17,18]$ or for fundamental studies of QED with trapped atomic ions [19-22]. Here, we first prove that the self-homodyne method is equivalent to the ideal configuration. Subsequently, we demonstrate that for increasing numerical aperture (NA) of an optical setup, the self-homodyne method approaches the Heisenberg limit faster than the methods employed in state-of-the-art optomechanical experiments.

\section{SELF-HOMODYNE DETECTION AND THE HEISENBERG LIMIT}

A schematic drawing of the self-homodyne setup is presented in Fig. 1. The mirror of radius $R_{S}$ and the detector of 
(a)

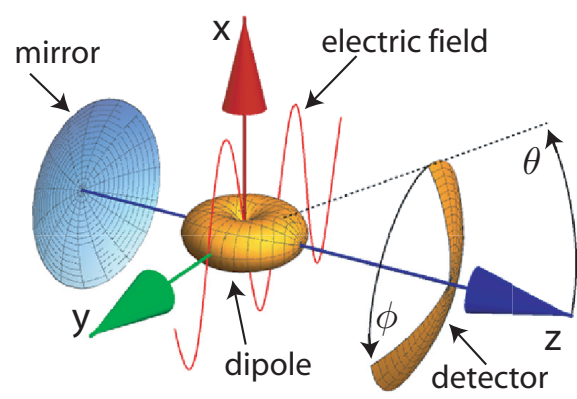

(b)

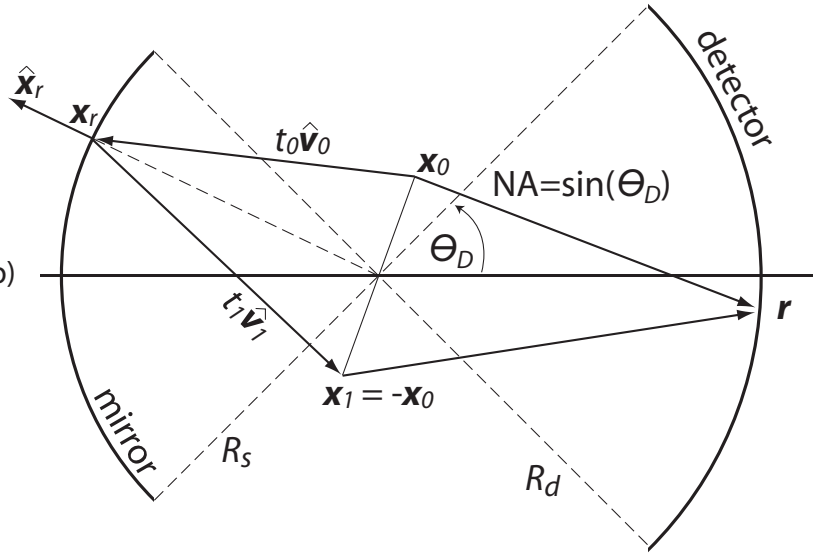

FIG. 1. Schematic representation of the self-homodyne setup. (a) Three-dimensional overview represented in both the Cartesian coordinate system and the spherical coordinate system used in this article. (b) Bidimensional section. A spherical mirror of radius $R_{s}$ images the light emitted by a dipolar scatterer located at $\boldsymbol{x}_{0}$ at the point $\boldsymbol{x}_{1}=-\boldsymbol{x}_{0}$. An optical path for the spherical wave fronts is drawn departing from $\boldsymbol{x}_{0}$, reflected at $\boldsymbol{x}_{r}$, and imaged at $\boldsymbol{x}_{1}$. The ray travels an optical path given by $t_{0}+t_{1}=2 R_{s}$ along the directions defined by the unit vectors (versors) $\hat{\boldsymbol{v}}_{0}$ and $\hat{\boldsymbol{v}}_{1}$. The light emitted by the scatterer interferes with the scatterer's image on the detector of radius $R_{d}$ at position $\boldsymbol{r}$. The detector collects all the light within the polar angle interval $\left[0, \theta_{D}\right]$ from the optical axis bisecting the mirror and the detector. The numerical aperture of the setup is defined as $\mathrm{NA}=\sin \theta_{D}$.

radius $R_{d}$ are concentric around the origin. A pointlike dipolar emitter of radiation with wavelength $\lambda$ is located at $\boldsymbol{x}_{0}$ near the origin. This configuration is referred to as a "half-cavity" [23]. We are interested in deducing the position $\boldsymbol{x}_{0}$ of the scatterer with respect to the origin of the coordinate system by measuring the light intensity impinging on the detector at different angles. For this discussion, we will refer to $\boldsymbol{x}_{0}$ both as the position or the displacement to be detected.

We assume that the scattered light is reflected and detected in the far field. This approximation corresponds to the condition under which the modulus of the displacement $\left\|x_{0}\right\|$ from the origin is much smaller than the distance $R_{d}$ between the particle and the detector and much smaller than the radius of curvature $R_{s}$ of the mirror. The far-field approximation allows us to consider the dipolar scatterer as a source of spherical waves whose differential radiated power $d p_{\text {dip }}$ per unit solid angle $d \Omega$ is given by [13]

$$
d p_{\text {dip }}=\frac{3}{8 \pi} P\left(1-|\hat{\boldsymbol{n}} \cdot \hat{\boldsymbol{\epsilon}}|^{2}\right) d \Omega,
$$

where $P$ is is the total power radiated, $\hat{\boldsymbol{n}}$ is given direction in space, and $\hat{\boldsymbol{\epsilon}}$ is the polarization of the driving light.

Under these conditions, we can model the light field's propagation and interference by using the Huygens-Fresnel integral in scalar form. Furthermore, the spherical optical elements enable us to solve the problem without using the paraxial approximation while disregarding the $1 / R$ factor in the Green's function of the Huygens-Fresnel integral.

At the location $\boldsymbol{r}=\hat{\boldsymbol{n}} R_{d}$ on the detector, the expression for the field's amplitude is

$$
E_{S}(\boldsymbol{r})=E_{0} \exp \left(i \frac{2 \pi}{\lambda} \hat{\boldsymbol{n}} \cdot \boldsymbol{x}_{0}\right)
$$

where $E_{0}$ is a constant complex amplitude. The amplitude $E_{0}$ contains the rescaling and phase prefactor $\exp \left(-i 2 \pi R_{d} / \lambda\right) / R_{d}$, which is constant across the detector surface. For a small displacement in any direction $\left(\left\|x_{0}\right\| \ll R_{S}\right)$, the mirror generates an image of the emitter at the opposite side of the origin, at $\boldsymbol{x}_{1}=-\boldsymbol{x}_{0}$ (see Appendix A). The polarization and wave fronts of the image are the same as for the primary dipole. The image field is phase referenced to the scattered field and can be expressed at the detector as

$$
E_{i}(\boldsymbol{r})=-\rho E_{0} \exp \left(-i \frac{2 \pi}{\lambda}\left(\hat{\boldsymbol{n}} \cdot \boldsymbol{x}_{0}+2 R_{s}\right)\right),
$$

where $\rho$ is the reflection coefficient for the electric field at the mirror. The minus sign in front accounts for the phase difference accumulated at the reflection.

The intensity measured at $\boldsymbol{r}$ is

$$
\begin{aligned}
I(\boldsymbol{r}) & \propto\left|E_{s}(\boldsymbol{r})+E_{i}(\boldsymbol{r})\right|^{2} \\
& \propto 1+\rho^{2}-2 \rho \cos \left(\frac{4 \pi}{\lambda}\left(\hat{\boldsymbol{n}} \cdot \boldsymbol{x}_{0}+R_{S}\right)\right) .
\end{aligned}
$$

We see that the detected light intensity at any position $\boldsymbol{r}$ is sinusoidal as a function of the position $\boldsymbol{x}_{0}$. To detect the position via an intensity modulation univocally, the modulus of the displacement must be subwavelength: $\left\|x_{0}\right\|<\lambda$, a condition normally fulfilled in experiments $[4,24]$.

We obtain the maximum sensitivity on the slope of the interference pattern, i.e., when the equality $4 \pi R_{s} / \lambda=2 \pi m \pm$ $\pi / 2$ holds, with $m$ a natural number. By simplifying Eq. (4) to first order in $\left\|\boldsymbol{x}_{0}\right\| / \lambda$ under these conditions, we obtain

$$
I(\boldsymbol{r}) \propto 1+\rho^{2} \pm \rho \frac{8 \pi}{\lambda}\left(\hat{\boldsymbol{n}} \cdot \boldsymbol{x}_{0}\right) .
$$

In this formula, either sign in the last term leads to the same sensitivity. For simplicity, we select the positive sign.

To impose a stable phase of interference for maximal motion sensitivity, the experimenter can take advantage of the mass difference between the scatterer and the mirror. As already demonstrated in Refs. [19,22], the oscillations of the particle are usually much faster than the drifts of the mirror position. By averaging the detected light over several periods of the particle's oscillation, one can generate a feedback signal to compensate for the mirror movements without compromising the information obtained about the particle's motion. 
Equation (5) can be compared to the expression for the intensity of the ideal homodyne scheme, adapted from Ref. [14]:

$$
I_{\text {ideal }}(\boldsymbol{r}) \propto 1+\gamma^{2}+\gamma \frac{4 \pi}{\lambda}\left(\hat{\boldsymbol{n}} \cdot \boldsymbol{x}_{0}\right) .
$$

Here, $\gamma$ is the proportionality coefficient between the reference electric field and the scattered electric field. We see that $\gamma$ substitutes for the reflection coefficient $\rho$.

The experimenter's control over the power of the reference field is manifested in the coefficient $\gamma$. In state-of-the-art experiments adopting homodyne detection schemes [7,25], this control allows one to work in a regime in which $\gamma^{2}+1 \sim \gamma^{2}$, corresponding to adjusting the intensity of the reference field to be much stronger than that of the direct field. However, this regime is not accessible for the self-homodyne method because $\rho$ is bounded by the condition $\rho \leqslant 1$. Furthermore, we note that the position-dependent terms in Eqs. (5) and (6) differ by a factor of 2 . The discrepancy stems from the fact that, in contrast to the ideal detection configuration, the reference field source, which is the image created by the mirror, is not fixed to the center of the curvature of the mirror. The displacement of the particle causes a shift of the image in the opposite direction relative to the center of the curvature, thus increasing the sensitivity by a factor of 2 . Also in contrast to the ideal case, in the self-homodyne method the half solid angle occupied by the mirror cannot be used for detection and the interference is observed only on the opposite half of the solid angle. In the remainder of this section, we calculate the limit for position detection to show that the self-homodyne setup can be considered equivalent to the ideal configuration notwithstanding these differences. In Appendix B, we expand our discussion of the self-homodyne process to highlight the directionality of the interference effect and its connection to the spontaneous emission of an atomic scatterer-mirror system for a deeper understanding of the physical phenomenon.

Since the mirror is in the far field, the polarization of the image is the same as the polarization of the original dipole. Combining the interference term and the angular distribution of the scattered radiation, we obtain the differential detected power $d p_{\text {det }}$ :

$$
d p_{\text {det }}=\left(1+\rho^{2}+\rho \frac{8 \pi}{\lambda}\left(\hat{\boldsymbol{n}} \cdot \boldsymbol{x}_{0}\right)\right) d p_{\text {dip }} .
$$

Any displacement $\boldsymbol{x}_{0}$ causes an intensity modulation proportional to

$$
d \beta=\frac{8 \pi \rho}{\lambda}\left(\hat{\boldsymbol{n}} \cdot \hat{\boldsymbol{x}}_{0}\right) d p_{\mathrm{dip}},
$$

where $\hat{\boldsymbol{x}}_{0}$ is the unit vector $\hat{\boldsymbol{x}}_{0}=\boldsymbol{x}_{0} /\left\|\boldsymbol{x}_{0}\right\|$. The sensitivity to position per unit solid angle, $d \beta$, combines the modulations due to the homodyning field and the distribution of the scattered light. For small absolute displacements $\left\|x_{0}\right\| \ll \lambda$, the power $d p_{\text {det }}$ described by Eq. (7) is dominated by the term $\rho^{2}+1$. This constant bias in the signal determines the power spectral density of the fluctuations $d \sigma$ via the shot noise:

$$
d \sigma=\frac{\hbar c}{\lambda}(R+1) d p_{\text {dip }},
$$

where $R=\rho^{2}$ is the reflection coefficient of the mirror for light intensity. The imprecision in detecting the position $\boldsymbol{x}_{0}$ under a solid angle $d \Omega$ is defined as [14]

$$
s\left(\hat{\boldsymbol{x}}_{0}\right)=\frac{d \sigma}{d \beta^{2}}=\frac{\hbar \lambda c(R+1)}{64 \pi^{2} R\left(\hat{\boldsymbol{n}} \cdot \hat{\boldsymbol{x}}_{0}\right)^{2} d p_{\mathrm{dip}}} .
$$

The imprecision captures the interplay between the modulation of the homodyne interference and the radiated power. In an optomechanics experiment, the imprecision corresponds to the noise floor of the detected motional spectrum. Decreasing the imprecision increases the visibility of the thermal mechanical peak of the particle oscillator above the detection noise [1]. In the context of feedback cooling [26], a lower imprecision in the detection of motion is beneficial for reducing the thermal phonon occupation number. The total imprecision over a detector area $D$ is defined as [14]

$$
S_{D}\left(\hat{\boldsymbol{x}}_{0}\right)=\left(\int_{D} s\left(\hat{\boldsymbol{x}}_{0}\right)^{-1}\right)^{-1} .
$$

Repeating the calculation from Eq. (7) to Eq. (10) for a dipole scatterer measured with the ideal homodyne configuration, we obtain

$$
\frac{s^{\text {ideal }}\left(\hat{\boldsymbol{x}}_{0}\right)}{s\left(\hat{\boldsymbol{x}}_{0}\right)}=\frac{4 R}{1+R} .
$$

Due to the disparity in the solid angle available for detection, the ratio of total imprecision in the two schemes is only half of the value in Eq. (12):

$$
\frac{S_{\Omega}^{\text {ideal }}\left(\hat{\boldsymbol{x}}_{0}\right)}{S_{\Omega / 2}\left(\hat{\boldsymbol{x}}_{0}\right)}=\frac{2 R}{1+R} .
$$

Since mirrors with reflectivity $>0.99$ are available on the market, we will assume $R \sim 1$ in the remainder of this work. This result holds for any orientation of the half-cavity and shows that for very high mirror reflectivity, the half-cavity scheme has the same total imprecision as the ideal configuration in any displacement direction of the dipolar emitter. Thus, we see that the half-cavity setup is a practical implementation of the ideal theoretical configuration and, like the ideal configuration, is limited only by the back action. Note that displacements along different axes are not necessarily detected with the same imprecision. For example, for linearly polarized dipoles, the lowest imprecision $S_{\min }=$ $5 \hbar c \lambda /\left(32 \pi^{2} P\right)$ is achieved for scatterer displacements orthogonal to the polarization direction, which can be compared with the maximal imprecision $S_{\max }=5 \hbar c \lambda /\left(16 \pi^{2} P\right)$ corresponding to displacements along the polarization axis.

\section{SETUP WITH LIMITED NA AND A QUADRANT DETECTOR}

In typical levitated optomechanics experiments [4], the scatterer is a $\mathrm{SiO}_{2}$ nanoparticle that oscillates in a harmonic potential generated by optical tweezers or a Paul trap. Each particle is composed of $\sim 10^{9}$ atoms and has a radius of $\sim 100 \mathrm{~nm}$. The trapping frequencies of such particles are $10^{2}-10^{5} \mathrm{~Hz}$, and the amplitudes of single quanta of oscillation of the particles are $\left\|\boldsymbol{x}_{0}\right\| \sim 10-100 \mathrm{pm}$. These excursions are much smaller than the wavelength $\lambda \sim 500-1500 \mathrm{~nm}$ of the laser field that illuminates the scatterer, validating the assumption we made in the previous section. 


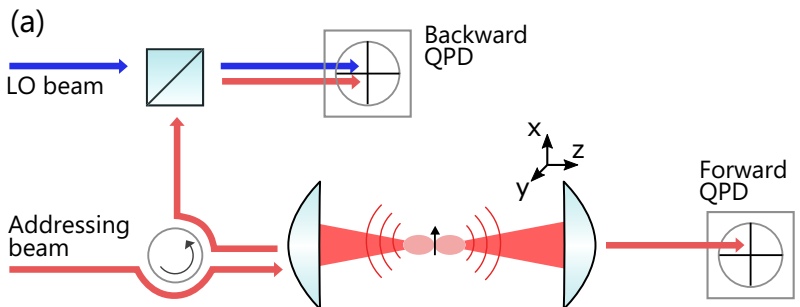

(b)

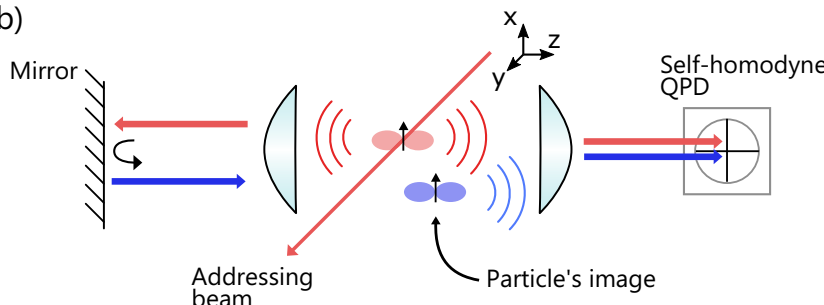

FIG. 2. Comparison of the forward and backward techniques with the self-homodyne method. (a) Forward and backward techniques. A Gaussian beam propagating along the $z$ axis is focused on the nanoparticle. The scattered light from the particle is detected in the forward and backward directions on quadrant photodetectors (QPDs) to measure the scatterer's position. (b) Self-homodyne technique. The half-cavity axis is aligned with the $z$ axis, and the particle is illuminated by a beam propagating along the $y$ axis. In the main text, we compare the techniques under the assumption that the polarization of the incident light field is aligned with the $x$ axis.

The particle's position is observed via homodyne detection by locating the scatterer between a pair of confocal lenses $[27,28]$. The first lens focuses an illuminating Gaussian beam on the particle, and the second lens collimates the scattered light and the incident beam, which then impinge on a detector. In this way, the Gaussian beam is the source for the scattered light and can be used as the reference field for homodyning. This technique is referred to as the "forward" detection method. Alternatively, the backscattered light from the particle can be used to infer the scatterer's position [5]. In this case, the returning light is not lost but collimated by the lens that focuses the Gaussian beam onto the nanoparticle. The returning field is interfered with a reference beam for the analysis of the scatterer's position. This technique is called the "backward" detection method.

We present in Fig. 2 a schematic overview of forward, backward, and self-homodyne setups to illustrate the configurations that are the basis for the comparison. These three detection methods are not mutually exclusive. For example, the forward and backward methods can be used together, as depicted in Fig. 2(a), with the optical axis oriented along the $y$ axis of Fig. 2(b). Such combined configurations have not been explored in experiments due to the increased complexity in the optical and electronic setups. Therefore, in the main text, we compare experiments implementing only one technique at a time. In Appendix E, we compare the combined forward-backward method with the self-homodyne method.

The self-homodyne approach has three advantages compared to the forward and backward setups. First, the mirror generates a secondary dipole image that matches the wave fronts of the primary field, while the Gaussian illuminating beam of the forward technique does not match the wave fronts of the light emitted by the dipolar scatterer. Second, in the self-homodyne method, the coupled system of the mirror and the scatterer emits light in only half of the solid angle. In contrast, both the forward and backward methods may analyze only half of the emitted light scattered by the particle over the entire solid angle. Third, unlike in the forward and backward detection schemes, the optical axis of the half-cavity may have arbitrary orientation with respect to the illumination beam. In this section, we will show how to make use of the three advantages of self-homodyning to obtain a lower imprecision in the measurement of the scatterer's position than is achievable by means of the forward and backward techniques. We consider a possible implementation adopting limited-NA optical elements and a quadrant photodetector.

Similarly to the forward and backward methods, the self-homodyne method can be implemented by locating the scatterer between a pair of confocal lenses in order to use a flat mirror and a flat detector, as demonstrated in Refs. [21,22]. The lenses introduce a cut over the solid angle, which can be modeled by reducing the effective NA of the optical spherical components, as already suggested in Fig. 1.

Three directions describe the setup: the versor $\hat{z}$ pointing from the mirror to the detector of the half-cavity system, the displacement of the scatterer $\hat{\boldsymbol{x}}_{0}$, and the polarization $\hat{\epsilon}$ of the illuminating beam. For the description of directions and positions in space, we will make use of angular and Cartesian notation according to the map $(x, y, z)=$ $r(\sin \theta \cos \phi, \sin \theta \sin \phi, \cos \theta)$ as depicted in Fig. 1(a). We set the axis of the half-cavity along the $z$ axis so that the detector $D$ is located between the polar angles $0 \leqslant \theta \leqslant \theta_{D}$ at any azimuth $0 \leqslant \phi<2 \pi$. The direction $\hat{\boldsymbol{x}}_{0}$ is indicated by the polar angle $\theta_{0}$ and the azimuthal angle $\phi_{0}$, and the polarization $\hat{\boldsymbol{\epsilon}}$ by the angles $\theta_{\epsilon}$ and $\phi_{\epsilon}$. This configuration can be interpreted in terms of numerical aperture via the expression $\mathrm{NA}=\sin \theta_{D}$. A script in Wolfram Mathematica v. $11.3 \mathrm{cal}-$ culates the imprecision for arbitrary angles $\theta_{0}, \phi_{0}, \theta_{\epsilon}, \phi_{\epsilon}$, and $\theta_{D}$. The solution is cumbersome but useful to evaluate specific experimental implementations where different constraints are present or particular optima of operation are desired. Here, we have decided to focus on the configuration that obtains minimum differential imprecision for a symmetric lens setup, assuming linearly polarized light.

In general, a superposition of circular or linear polarizations can be used to illuminate the nanoparticle. However, to minimize the imprecision, linear polarization is preferred because the distribution of scattered light is less homogeneous over the solid angle, thus allowing higher intensity to be directed onto a limited-NA detector. In addition, circularly polarized light provides a torque, causing uncontrolled spinning of the levitated nanoparticles [29,30]. We include the discussion of arbitrary polarization to Appendix $\mathrm{C}$ because it is relevant for nonlinear dipolar scatterers such as isolated atoms or molecules.

The configuration with which the minimum differential imprecision $s$ is obtained is found by minimizing Eq. (10). We note that the minimization of $s$ with respect to the displacement direction $\hat{\boldsymbol{x}}_{0}$ is independent from the 
minimization over the light polarization $\hat{\boldsymbol{\epsilon}}$. The lowest differential imprecision is found for $\theta_{\epsilon}=\pi / 2$ and $\phi_{\epsilon}=0$, corresponding to polarization along the $x$ axis, and for $\theta_{0}=0$, corresponding to displacement along the $z$ axis. The propagation direction of the illuminating beam is fixed in the $y z$ plane by the minimization of $s$. For simplicity, we set the beam orientation to be along the $y$ axis. In this configuration, the total imprecision as a function of the detector aperture $\theta_{D}$ is

$$
S_{l}=\frac{20 \hbar c \lambda}{P \pi^{2}\left[128-90 \cos \left(\theta_{D}\right)-35 \cos \left(3 \theta_{D}\right)-3 \cos \left(5 \theta_{D}\right)\right]} .
$$

In state-of-the-art experiments, large sections of the solid angle are collimated by a lens on the small area of a single detector [19] or a quadrant photodiode [31,32]. In selfhomodyne detection, the intensity common to all quadrants depends on the proximity of the scatterer to the detector, and the modulations between pairs of different pixels are caused by displacements of the scatterer in directions orthogonal to the light propagation [27]. With a discrete pixel detector, the imprecision must be calculated as the weighted mean over the available detector area [14] rather than by the integral of Eq. (11) as we describe in Appendix D.

We finally have the tools in hand to compare the forward, backward, and self-homodyne methods. In this comparison, we align the optical axis of the forward and backward detection along the $z$ axis and the light is linearly polarized along the $x$ axis. For each technique, we calculate the detection efficiency as the inverse of the imprecision in detecting the scatterer displacements along the $x, y$, and $z$ axes, and we normalize it with the maximum efficiency obtained at the Heisenberg limit achievable in the system: $S_{\min }^{-1}=$ $32 \pi^{2} P /(5 \hbar c \lambda)$. The efficiencies are calculated as being measured by an ideal quadrant photodetector with unit quantum efficiency. The latter is oriented with its quadrants being divided by the $x$ and $y$ axes.

Figure 3 shows the detection efficiencies of the three techniques as a function of the optical NA of the confocal lenses. The horizontal lines mark the Heisenberg limits for displacements in the $y z$ plane and along the $x$ axis. Comparing data for a given axis, the half-cavity shows higher efficiency than the forward or backward detection. We attribute the improvement to both the concentration of the information in only half of the solid angle and the match of polarization and wave fronts between the reference and the scattered light fields. In Fig. 3 we also plot the efficiency corresponding to the ideal pixel detector described by Eq. (14). A comparison of the ideal detector with the discrete pixel detector, in both cases used in a self-homodyne measurement of displacement along the $z$ axis, reveals that the imprecisions corresponding to the two detectors are equivalent up to NA $\sim 0.8$. Thus, we see that the adoption of a quadrant detector is not detrimental for practical implementations aiming to reach the lowest possible imprecision at NA $<0.8$. Further comparisons between the detection efficiencies for displacements along the principal axes achievable with a quadrant detector and with an ideal detector are presented in Appendix D. Finally, we mention that the freedom of choice in the orientation of the half-

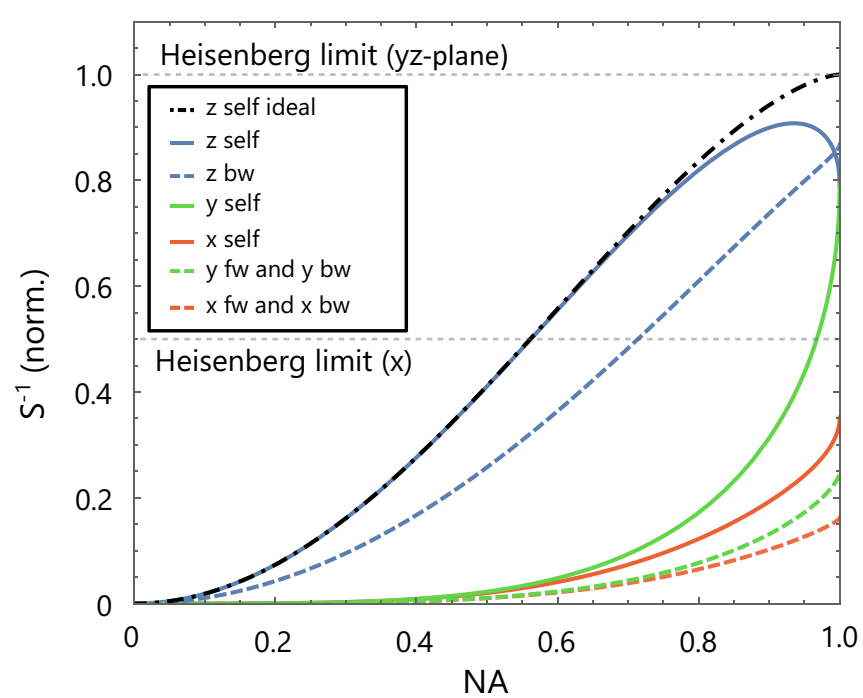

FIG. 3. Comparison of detection efficiency between the halfcavity setup (self) and the forward $(f w)$ and backward $(b w)$ detection schemes as a function of the NA. The labels $x, y$, and $z$ refer to specific motional axes of the scatterer. The forward and backward imprecisions are plotted with dashed colored lines (adapted from [14]), and the self-homodyne imprecisions with solid colored lines. The legend's entries are sorted according to the heights of the curves at NA $=0.9$. The lines $f w$ and $b w$ for the $y$ and $x$ axes overlap. Along the $x$ and $z$ axes, the data obtained with the forward scheme are equal to those obtained with the backward scheme. The efficiency has been normalized for the maximum efficiency at the Heisenberg limit $S_{\min }^{-1}=32 \pi^{2} P /(5 \hbar c \lambda)$. The efficiency for $z$ displacements for the forward method is zero in the selected configuration, and it is not reported in the plot.

cavity optical axis can further be exploited beyond the current configuration, for example, to achieve the imprecision that is shown in Fig. 3 for the half-cavity setup along the $z$ axis along any direction in the $y z$ plane.

Further considerations on the applicability of the technique concerning the limits imposed by the Rayleigh approximation and the photon sensitivity of the detector are presented in Appendices F and G.

\section{CONCLUSION}

The self-homodyne detection method implements an ideal setup that is capable of detecting the motion of a dipolar scatterer at the Heisenberg limit. The signal is obtained via self-referencing the scattered light by using a spherical mirror or a combination of a lens and a flat mirror to reflect the light back in the direction of the scatterer. We have analyzed the setup at limited NA for a possible realization that combines two confocal lenses, a flat mirror, and a quadrant detector. We have discussed the orientation of the setup that would minimize the imprecision in detecting the scatterer position and that can achieve higher efficiency than the state-of-theart forward and backward detection methods. Compared to the forward and backward schemes, the mirror generates an ideal reference field and allows for arbitrary orientation of the mirror-detector axis for optimal reconstruction of the scatterer's position by adopting a single one-sided detector. 
Compared to an optical implementation with a deep parabolic mirror, our method offers a scalable approach for detection that can be implemented in experiments with limited optical access.

As discussed in Appendix C, self-homodyne can also be used to detect the position and motion of single-photon resonant scatterers [33] reemitting light with circular polarization. These emitters can be used to study the influence of a reflective boundary condition on isolated quantum systems, which can be measured as a force that affects the scatters' position and motion $[23,34]$.

The arbitrary orientation of the half-cavity with respect to the illumination beam constitutes an appealing option for position detection in complex experimental setups. For example, the self-homodyne apparatus could be mounted orthogonally to an optical cavity or to a forward-backward apparatus. Furthermore, the high sensitivity of the half-cavity setup for the detection of motion at low NAs could be beneficial in levitated optomechanics systems with limited optical access, such as electromagnetic or magneto-gravitational traps.

\section{ACKNOWLEDGMENTS}

We would like to thank Gabriel Araneda for detailed discussions and suggestions regarding the experimental setup. We thank Lukas Novotny for suggesting the connection between our method and previous microscopy applications and Markus Sondermann for indicating to us the studies of dipolar fields in deep parabolic mirrors. G.C. would like to thank Ruggero Caravita for inspiring discussions about isolated systems and about the Wigner-Eckart theorem. This work has received funding from the European Unions Horizon 2020 research and innovation programme under the Marie Skodowska-Curie Grant Agreement No. 801110 and the Austrian Federal Ministry of Education, Science and Research (BMBWF). It reflects only the authors' view; the EU agency is not responsible for any use that may be made of the information it contains. This work was also supported by the Austrian Science Fund (FWF) Project No. Y951, by the ESQ Discovery grant "Sympathetic detection and cooling of nanoparticles levitated in a Paul trap" of the Austrian Academy of Sciences, and by the Institut für Quanteninformation $\mathrm{GmbH}$.

\section{APPENDIX A: POSITION OF THE IMAGE}

In this Appendix, we derive that a hemispherical mirror produces a secondary image of a pointlike spherical wave emitter on the opposite side of its curvature center. We remind the reader that the displacement of the emitter from the origin of the half-cavity is small enough that the light field propagation can be described by using the Huygen-Fresnel integral. Under this assumption, we model the dipolar scatterer as an emitter of spherical waves. We will show that the mirror generates an image at $\boldsymbol{x}_{1}=-\boldsymbol{x}_{0}$ by demonstrating that the optical path between $\boldsymbol{x}_{1}$ and $\boldsymbol{x}_{0}$ is the same for any part of the outgoing spherical wave fronts which intersect the mirror.

With reference to Fig. 1, from $x_{0}$, we can follow the wave front along the generic direction described by the versor $\hat{\boldsymbol{v}}_{0}$ $\left(\left\|\hat{\boldsymbol{v}}_{0}\right\|=1\right)$ until encountering the surface of the sphere of radius $R_{S}$ at $\boldsymbol{x}_{r}$. There, the wave front is locally reflected and the light arrives at $\boldsymbol{x}_{1}$ following the direction of the versor $\hat{\boldsymbol{v}}_{1}$. The optical path length between $\boldsymbol{x}_{0}$ and $\boldsymbol{x}_{r}$ is $t_{0}$ and between $\boldsymbol{x}_{r}$ and $\boldsymbol{x}_{1}$ is $t_{1}$. For any generic direction $\hat{\boldsymbol{v}}_{0}$ and length $t_{1}$ the point $\boldsymbol{x}_{1}$ can be found by solving the following system of equations:

$$
\begin{gathered}
\boldsymbol{x}_{r}=\boldsymbol{x}_{0}+\hat{\boldsymbol{v}}_{0} t_{0}, \\
\left\|\boldsymbol{x}_{r}\right\|^{2}=R_{s}, \\
\boldsymbol{x}_{1}=\boldsymbol{x}_{r}+\hat{\boldsymbol{v}}_{1} t_{1}, \\
\hat{\boldsymbol{v}}_{1}=\hat{\boldsymbol{v}}_{0}-2\left(\hat{\boldsymbol{v}}_{0} \cdot \hat{\boldsymbol{x}}_{r}\right) \hat{\boldsymbol{x}}_{r},
\end{gathered}
$$

where $\hat{\boldsymbol{x}}_{r}=\boldsymbol{x}_{r} /\left\|\boldsymbol{x}_{r}\right\|^{2}$.

The scalar product of Eq. (A1) with $\hat{\boldsymbol{v}}_{0}$ allows us to find the following relation for $t_{0}$ :

$$
t_{0}=-\left(\hat{\boldsymbol{v}}_{0} \cdot \boldsymbol{x}_{0}\right)+R_{s}\left(\hat{\boldsymbol{v}}_{0} \cdot \hat{\boldsymbol{x}}_{r}\right) .
$$

Then, we consider the square norm of Eq. (A1):

$$
R_{s}^{2}=\left\|\boldsymbol{x}_{0}\right\|^{2}+t_{0}^{2}+2\left(\hat{\boldsymbol{v}}_{0} \cdot \hat{\boldsymbol{x}}_{0}\right) t_{0}
$$

By combining Eq. (A5) and Eq. (A6), we obtain

$$
\left(\hat{\boldsymbol{v}}_{0} \cdot \hat{\boldsymbol{x}}_{r}\right)=\sqrt{1-\frac{\left\|\boldsymbol{x}_{0}\right\|^{2}-\left(\hat{\boldsymbol{v}}_{0} \cdot \boldsymbol{x}_{0}\right)^{2}}{R_{s}^{2}}} .
$$

This relation reduces to $\left(\hat{\boldsymbol{v}}_{0} \cdot \hat{\boldsymbol{x}}_{r}\right)=1$ to first order in $\left\|\boldsymbol{x}_{0}\right\| / R_{s}$. In this approximation, we find that Eq. (A4) reduces to $\hat{\boldsymbol{v}}_{0}=$ $\hat{\boldsymbol{x}}_{r}=-\hat{\boldsymbol{v}}_{1}$.

With this simplification, we are now ready to evaluate $\boldsymbol{x}_{0}+$ $\boldsymbol{x}_{1}$ by combining Eqs. (A1) and (A3):

$$
\boldsymbol{x}_{0}+\boldsymbol{x}_{1}=\hat{\boldsymbol{x}}_{r}\left(2 R_{s}-t_{0}-t_{1}\right) .
$$

We see that the right-hand side of this expression is zero if $t_{0}+t_{1}=2 R_{s}$. This solution describes an image formed at $\boldsymbol{x}_{1}=-\boldsymbol{x}_{0}$ because it is valid for any versor $\hat{\boldsymbol{v}}_{0}$. The image is formed because $\boldsymbol{x}_{1}$ is the point at which the wave fronts interfere with the same phase delay. Thus, a pointlike light source located near the origin is imaged on the opposite side of the origin with a constant phase delay proportional to twice the radius of the hemisphere.

\section{APPENDIX B: MODEL DESCRIPTION}

In the self-homodyne method, the interference of the directly scattered and image fields can be interpreted as a variation of the spontaneous emission rate of the dipolar emitter. This variation in half-cavities has already been described in the literature for a perfectly conducting mirror $(\rho=1)$ by quantizing the electromagnetic field modes in the presence of a spherical mirror [35]. In this section, we arrive at the same results by taking into account the interference between the direct field and the reflected field, and we extend the results of the previous calculation to arbitrary mirror reflectivity.

We compare the emitted radiation in the presence of the mirror with the emitted radiation in free space. In the presence of the mirror, we divide the solid angle into three regions:

(1) $M$, the angular domain of the mirror,

(2) $\bar{M}$, the symmetric domain to $M$ obtained by reflection through the origin, and

(3) $F=4 \pi-M-\bar{M}$, the remaining domain. 
The ratio of the modified scattering rate $\gamma$ to the free space scattering rate $\gamma_{0}$ can be calculated as the ratio of emitted powers in the configurations with and without mirror. The ratio references the light exiting at all solid angles from the scatterer-mirror composite system to the light emitted by the scatterer in free space. The ratio is given by

$$
\frac{\gamma}{\gamma_{0}}=\frac{P(M)+P(\bar{M})+P(F)}{P}
$$

where $P(D)$ denotes the power radiated in the solid angle $D$ in the presence of the mirror and $P$ has already been introduced in Eq. (1). The radiated powers $P(D)$ are

$$
\begin{gathered}
P(F)=\int_{F} d p_{\mathrm{dip}}, \\
P(M)=\int_{M} T d p_{\mathrm{dip}}, \\
P(\bar{M})=\int_{\bar{M}}\left(1+\rho^{2}-2 \rho \cos [\omega \alpha(\hat{\boldsymbol{n}})]\right) d p_{\mathrm{dip}},
\end{gathered}
$$

where $T$ is the transmission coefficient for light intensity. For brevity, we adopt $\alpha(\hat{\boldsymbol{n}})=\left[\frac{2}{c}\left(\hat{\boldsymbol{n}} \cdot \boldsymbol{x}_{0}+R_{s}\right)\right]$ and $\omega=2 \pi c / \lambda$. Here, $\rho$ and $T$ can be generic functions of the direction $\hat{\boldsymbol{n}}$, but in any direction, $\rho(\hat{\boldsymbol{n}})^{2}+T(\hat{\boldsymbol{n}})=1$, that is, we assume the mirror is lossless. Since for any direction in $M$ there is an opposite in $\bar{M}$, we arrive at

$$
\frac{\gamma}{\gamma_{0}}=1-\frac{3}{4 \pi} \int_{\bar{M}} \rho(-\hat{\boldsymbol{n}})\left(1-|\hat{\boldsymbol{\epsilon}} \cdot \hat{\boldsymbol{n}}|^{2}\right) \cos [\omega \alpha(\hat{\boldsymbol{n}})] d \Omega .
$$

In Eq. (B5), the minus sign in the argument of the $\rho$ function reminds us of the correct reflectivity coefficient while integrating in the $\bar{M}$ region. As already pointed out in Ref. [35], this result indicates that the spontaneous emission of the dipolar emitter can be completely suppressed or doubled in the center of curvature of a hemispherical mirror. Considering the radiated power, we can interpret the underlying mechanism of the self-homodyne scheme in the following way. Assuming perfect reflection, each photon emitted by the scatterer is self-referenced by the mirror, with the result being a position-dependent enhancement or suppression of the spontaneous emission of the dipolar scatterer. Every photon that contributes to the scatterer back action can only be emitted by the scatterer-mirror system into the open half of the solid angle, and no information is lost on the side of the mirror.

The variation of spontaneous emission leads to a modulation of the dipole strength, causing a shift of the energy levels of the dipole. Applying the Kramers-Kronig formula to Eq. (B5) and approximating the result for a small shift compared to the carrier frequency, we obtain the formula for the energy shift $\Delta \omega$ already found in [35]:

$$
\frac{\Delta \omega}{\gamma_{0}}=-\frac{3}{8 \pi} \int_{\bar{M}} \rho(-\hat{\boldsymbol{n}})\left(1-|\hat{\boldsymbol{\epsilon}} \cdot \hat{\boldsymbol{n}}|^{2}\right) \sin [\omega \alpha(\hat{\boldsymbol{n}})] d \Omega .
$$

Compared to the derivation of Eqs. (B5) and (B6) previously reported in Ref. [35], our approach identifies the coefficient $\rho$ as the reflection coefficient for the electric field of the mirror.

\section{APPENDIX C: ATOMIC DIPOLE TRANSITION}

The self-homodyne technique can be used to detect the position of an atom. This was demonstrated experimentally for motion along the mirror-detector axis in Refs. $[19,20,22,36]$. Typically, atoms are driven at a frequency close to resonance with a transition between a ground state and an excited state in order to generate fluorescence. Under these conditions, the dipolar response of the atom is nonlinear. Unlike a linear dipole, an atom reemits radiation with both linear and circular polarization regardless of the driving field. Linear polarization corresponds to $\pi$ transitions in which the magnetic quantum number $m$ is preserved. Circular polarization corresponds to $\sigma$ transitions, i.e., the transition is characterized by a change in quantum number $\Delta m= \pm 1$.

The light emitted during both $\pi$ and $\sigma$ transitions can be used to detect the position of the atom in a self-homodyne setup. Position detection based on $\pi$ transitions is described in the main text. It is equivalent to detection based on a linear dipolar scatterer driven with linearly polarized light. For compatibility with the result reported in the main text, the quantization axis in an atomic experiment should be aligned along the $x$ axis of Figs. 1 and 2 in the main text. In this configuration, in Eq. (1), $\pi$ transitions correspond to light polarized along the axis $\hat{\boldsymbol{\epsilon}}=\hat{\boldsymbol{x}}$ and $\sigma$ transitions to light polarized along the axis combination described by $\hat{\boldsymbol{\epsilon}}_{ \pm}=(\hat{\boldsymbol{y}} \pm i \hat{z}) / \sqrt{2}$. The unpolarized decay of an atom, defined as the case in which we do not distinguish between the two transition types but integrate over the possible outcomes, is obtained by the replacement $\left(1-|\hat{\boldsymbol{n}} \cdot \hat{\boldsymbol{\epsilon}}|^{2}\right) \rightarrow 2 / 3$. The last formula can be found by applying the Wigner-Eckart theorem to a generic dipole transition.

We present in Fig. 4 the efficiencies for the unpolarized, $\pi$-polarized, and $\sigma$-polarized fluorescence of an atom as a function of the system NA. Here, $\sigma$-polarized refers to either $\sigma^{+}$or $\sigma^{-}$transitions that have the same efficiency. In the plot, we compare the detection efficiencies (inverse imprecisions) for atom displacements along the principal axes of the reference system. In the corresponding calculation, we consider an ideal pixel detector and not a quadrant detector. We see that the detection efficiency depends on the polarization of the scattered light, which is due to the different radiated power distributions for the $\pi$ and $\sigma$ transitions. The displacement of the atom can be detected with highest efficiency along the optical axis of the half-cavity. In this case, the light emitted due to $\pi$ transitions leads to a higher efficiency than what is achievable by observing $\sigma$-polarized fluorescence.

This difference between $\pi$ and $\sigma$ transitions can be tested in experiments by selecting photons based on polarization. The asymmetry can be measured by introducing an external magnetic field along the quantization axis ( $x$ axis). If a polarizing beam splitter is used to separate $y$-and $x$-polarized fluorescence, the radiation emitted during $\pi$ and $\sigma$ transitions is separated and can be analyzed independently [37]. The relevant formulas to reproduce Fig. 4 can be found in Ref. [38].

\section{APPENDIX D: QUADRANT DETECTOR}

In this Appendix, we describe in more detail the calculation with which we obtain the imprecision of the self-homodyne 


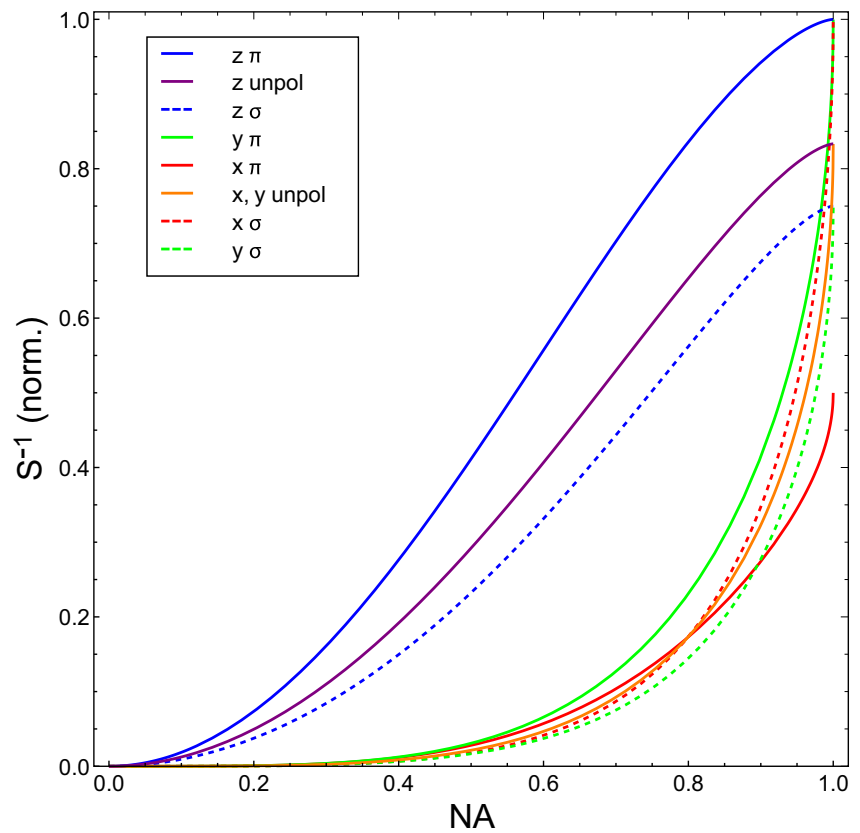

FIG. 4. Detection efficiencies for different displacements of an atomic dipole. The labels $\pi, \sigma$, and unpol (unpolarized) refer to the different angular distributions of emitted radiation as explained in the text. Each line is also labeled with $x, y$, and $z$ according to the axis of displacement of the atom. Unpolarized $x$ and $y$ efficiencies are identical. The values are normalized by $S_{\min }^{-1}=32 \pi^{2} P /(5 \hbar c \lambda)$ as done in the main text. The legend's entries are sorted according to the heights of the curves at NA $=0.7$.

technique as measured with a quadrant photodetector (QPD), and how it deviates from the idealized model of a differential pixel detector. This correction was already presented in Ref. [14] to model the forward and backward techniques, and we apply a similar analysis for the self-homodyne method. In the discussion, we assume the same configuration shown in Figs. 1 and 2, in which the QPD occupies the solidangle regions $Q$ contained in the polar-angle interval $0 \leqslant \theta$ $\leqslant \theta_{D}$. The quadrant regions $Q_{n}$ split the $Q$ region in the azimuthal-angle intervals $(n-1) \pi / 2 \leqslant \phi<n \pi / 2$, with $n=$ $\{1,2,3,4\}$. The total power incident on the $n$th quadrant is

$$
P^{Q_{n}}=\int_{Q_{n}}\left[1+\rho^{2}+\rho \frac{8 \pi}{\lambda}\left(\hat{\boldsymbol{n}} \cdot \boldsymbol{x}_{0}\right)\right] d p_{\text {dip }} .
$$

We reconstruct the particle's displacement $\boldsymbol{x}_{0}=\left(x_{0}, y_{0}, z_{0}\right)$ by taking advantage of the symmetries of the integrand. The combinations of the detected powers over the quadrants that we use to reconstruct the displacements are [14,27]

$$
\begin{aligned}
& x_{0}=\left[\left(P^{Q_{1}}+P^{Q_{4}}\right)-\left(P^{Q_{2}}+P^{Q_{3}}\right)\right] \frac{1}{4 B\left(\frac{\pi}{2}, 0\right)}, \\
& y_{0}=\left[\left(P^{Q_{1}}+P^{Q_{2}}\right)-\left(P^{Q_{3}}+P^{Q_{4}}\right)\right] \frac{1}{4 B\left(\frac{\pi}{2}, \frac{\pi}{2}\right)}, \text { and } \\
& z_{0}=\left[\sum_{n} P^{Q_{n}}-\int_{Q}\left(1+\rho^{2}\right) d p_{\text {dip }}\right] \frac{1}{4 B(0, \text { any })} .
\end{aligned}
$$

Here, $B\left(\theta_{0}, \phi_{0}\right)=\int_{Q_{n}} d \beta$ is the integral of the sensitivity to displacements along $\hat{\boldsymbol{x}}_{0}$ defined in Eq. (8) and is equal for

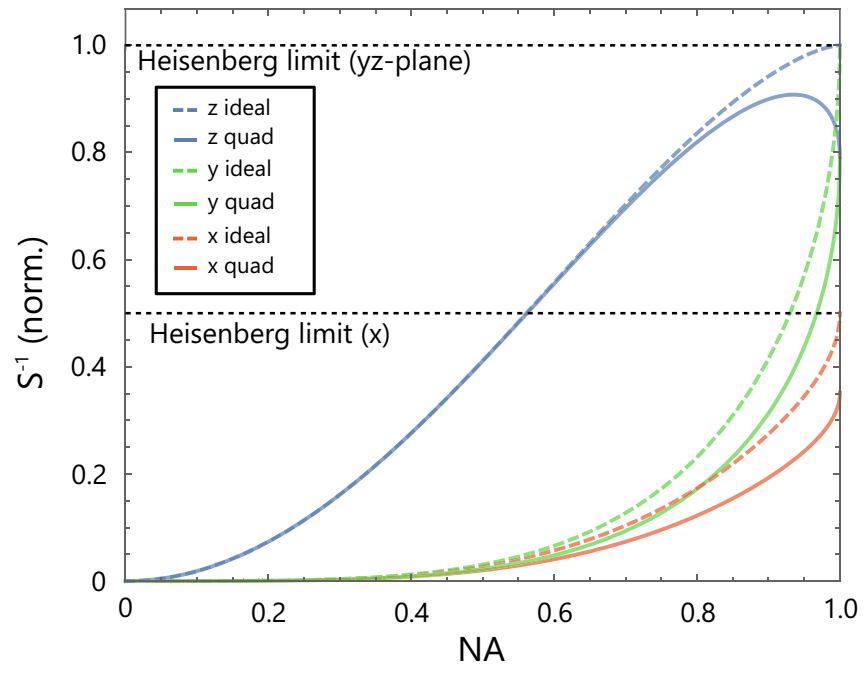

FIG. 5. Efficiencies of self-homodyne detection along three axes with an ideal differential pixel detector (dashed lines) and a quadrant photodetector (solid lines) as a function of the detector numerical aperture (NA). Values are normalized with respect to the efficiency at the Heisenberg limit for displacements in the $\phi_{0}=\pi / 2$ plane: $S_{\min }^{-1}=$ $32 \pi^{2} P /(5 \hbar c \lambda)$. The blue data, $z$ axis, was already plotted in Fig. 3 . The legend's entries are sorted according to the heights of the curves at $\mathrm{NA}=0.9$.

all quadrants thanks to the detector symmetry. The imprecision measured with the QPD is calculated as

$$
S_{\mathrm{QPD}}\left(\hat{\boldsymbol{x}}_{0}\right)=\frac{1}{16 B\left(\theta_{0}, \phi_{0}\right)^{2}} \int_{Q} \sigma d \Omega,
$$

where $\sigma$ is the spectral density of the power fluctuations defined in Eq. (9).

Figure 5 shows the detection efficiency $S_{\mathrm{QPD}}^{-1}$ of the selfhomodyne method obtained with the QPD, and $S_{D}^{-1}$, the detection efficiency obtained with the ideal pixel detec-

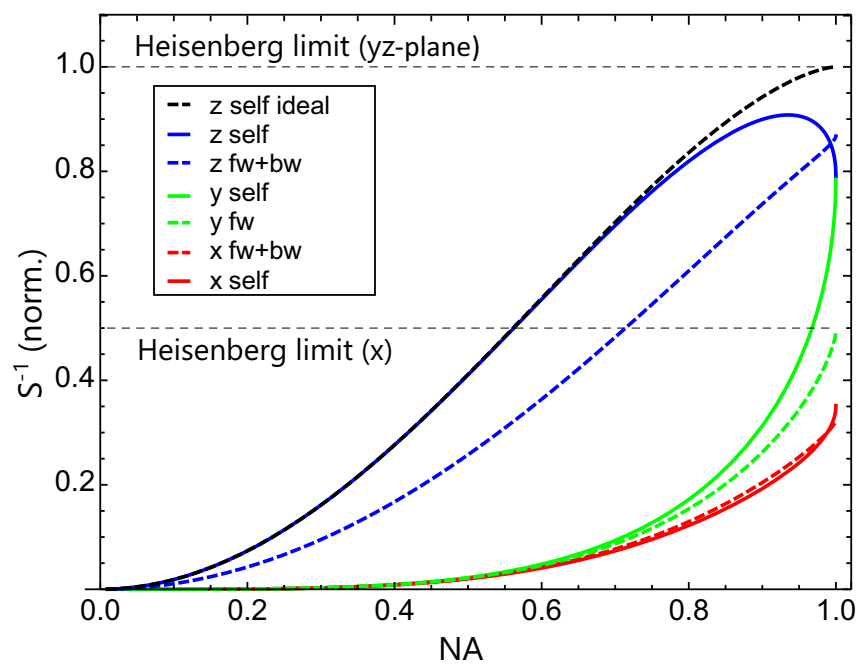

FIG. 6. Comparison of the detection efficiency of the combined backward and forward techniques $(f w+b w)$ with the self-homodyne method (self). The legend's entries are sorted according to the heights of the curves at NA $=0.9$. 


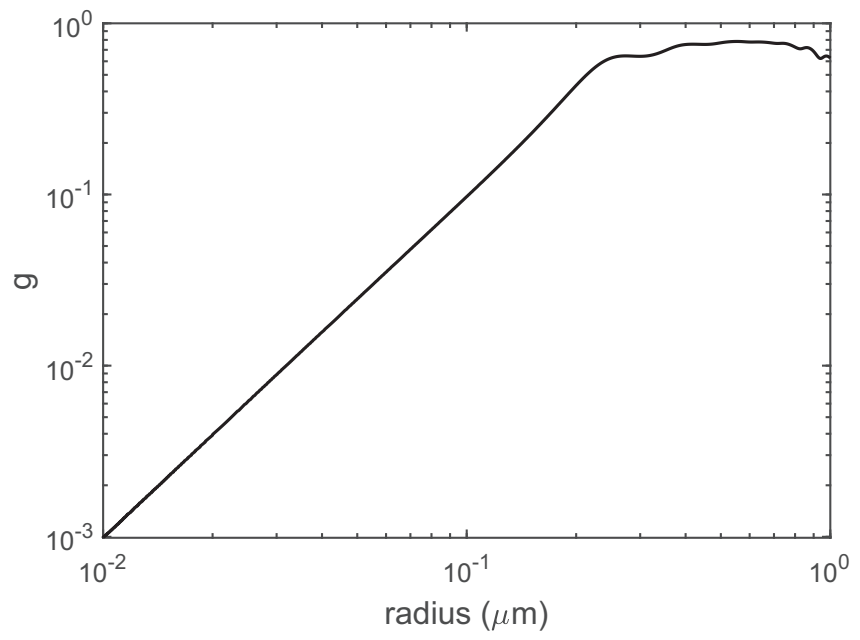

FIG. 7. Asymmetry parameter $g$ of the radiated power for an isolated dipole as a function of ${\mathrm{a} \mathrm{SiO}_{2}}_{2}$ nanosphere's radius. For the simulation, we considered a wavelength of the illuminating light of $\lambda=880 \mathrm{~nm}$ and a particle with index of refraction $n=1.45$.

tor (dashed lines), as a function of the detector numerical aperture $\mathrm{NA}=\sin \left(\theta_{D}\right)$. Values are normalized by $S_{\min }^{-1}=$ $32 \pi^{2} P /(5 \hbar c \lambda)$ as in the main text. The ideal efficiencies $S_{D}^{-1}$ are obtained by integrating Eq. (11) of the main text.

\section{APPENDIX E: COMBINED BACKWARD AND FORWARD}

In this Appendix, we compare the self-homodyne technique with the combined forward and backward techniques. Figure 6 presents the comparison with the configuration used for Fig. 3 in the main text.

\section{APPENDIX F: BEYOND THE RAYLEIGH APPROXIMATION}

Particles scatter light according to the dipolar pattern indicated by Eq. (1) if they are much smaller than the wavelength of the illuminating radiation $\lambda$. For particles comparable in size to $\lambda$, the scattering process should be modeled using Mie theory [13]. In this regime, particles smaller than the radiation wavelength scatter light predominantly in the forward direction. This forward-backward asymmetry affects the efficiency of self-homodyne detection because the mirror image of the dipolar scatterer has the opposite forward-backward asymmetry as a consequence of the reflection.

The asymmetry is quantified by the parameter $g$ [39]. We divide space into two regions separated by the $x z$ plane. If the scattering of the particle is entirely into the region $y>0$, then $g=1$; if it is into the region $y<0$, then $g=-1$; and if its pattern adheres to Rayleigh's formula, then $g=0$. Figure 7, which was generated using the software of Ref. [39], shows $g$ as a function of the radius of a $\mathrm{SiO}_{2}$ sphere for a wavelength of the illuminating field $\lambda=880 \mathrm{~nm}$. We see that the forward and backward powers differ by $10 \%$ if the radius of the sphere is about $100 \mathrm{~nm}$ and $1 \%$ if the radius is approximately $30 \mathrm{~nm}$. The asymmetry is lower if larger wavelengths were used. Furthermore, the effective asymmetry relevant for self-homodyne detection is reduced when the NAs of the optical components are taken into account. Also, the self-homodyne detection axis is orthogonal to the forward-backward axis, making the technique less sensitive to the emission asymmetry.

From these considerations, we conclude that there exist applications of the self-homodyne method in which the Mie-scattering correction is negligible and have therefore not been taken it into account in this work.

\section{APPENDIX G: PHOTODETECTION EFFICIENCY}

In homodyne experiments, the reference field may be much stronger than the field being studied, which leads to an amplification of the interference term over the shot noise of a real detector. By contrast, in the self-homodyne method primary and reflected fields have similar intensities. Thus, for ensuring a shot-noise limited detection, it is necessary to use high-efficiency detectors. As a practical example, we consider an experimental realization in which a silica particle with a radius of $75 \mathrm{~nm}$ is levitated at $1 \times 10^{-9}$ mbar. The self-homodyne detection is realized with an $800 \mathrm{~nm}$ laser, and we choose its intensity so that the particle scatters $300 \mathrm{nW}$ of light. With these settings, the back-action noise on the particle motion from photon recoil, $S_{\mathrm{BA}}=\frac{2}{5}(\hbar k / c) P$, is of the same order of magnitude as the noise induced by collisions with the background gas molecules, $S_{\mathrm{th}}=4 k_{\mathrm{B}} T \gamma m$, where $k_{\mathrm{B}}$ is the Boltzmann constant, $T$ is room temperature, $\gamma$ is the linewidth of the oscillation, and $m$ the particle's mass. Note that the Heisenberg limit occurs in the back-action dominated regime $\left(S_{\mathrm{BA}} \gg S_{\mathrm{th}}\right)$, so we use this example to determine minimal requirements for the detector's technical noise. By limiting our analysis to NA $=0.4$, the collected light impinging on the detector yields a shot-noise power spectral density of $\sim 1 \times 10^{-14} \mathrm{~W} / \sqrt{\mathrm{Hz}}$ according to Eq. (9), which is two orders of magnitude larger than the noise equivalent power of a typical commercially available avalanche photodiode, such as Thorlabs APD440A, operated with a $50 \mathrm{~s}$ averaging time. This example demonstrates that the self-homodyne could be implemented in an efficient way in a realistic levitated optomechanics setup.
[1] M. Aspelmeyer, T. J. Kippenberg, and F. Marquardt, Rev. Mod. Phys. 86, 1391 (2014).

[2] M. Carlesso, A. Bassi, M. Paternostro, and H. Ulbricht, New J. Phys. 21, 093052 (2019)

[3] M. Aspelmeyer, P. Meystre, and K. Schwab, Physics Today 65(7), 29 (2012)
[4] J. Millen, T. S. Monteiro, R. Pettit, and A. N. Vamivakas, Rep. Prog. Phys. 83, 026401 (2020).

[5] F. Tebbenjohanns, M. Frimmer, V. Jain, D. Windey, and L. Novotny, Phys. Rev. Lett. 124, 013603 (2020).

[6] U. Delić, M. Reisenbauer, K. Dare, D. Grass, V. Vuletić, N. Kiesel, and M. Aspelmeyer, Science 367, 892 (2020). 
[7] L. Magrini, P. Rosenzweig, C. Bach, A. Deutschmann-Olek, S. G. Hofer, S. Hong, N. Kiesel, A. Kugi, and M. Aspelmeyer, Nature (London) 595, 373 (2021).

[8] N. P. Bullier, A. Pontin, and P. F. Barker, Rev. Sci. Instrum. 90, 093201 (2019).

[9] G. Ranjit, D. P. Atherton, J. H. Stutz, M. Cunningham, and A. A. Geraci, Phys. Rev. A 91, 051805(R) (2015).

[10] J. Gieseler, B. Deutsch, R. Quidant, and L. Novotny, Phys. Rev. Lett. 109, 103603 (2012).

[11] V. Jain, J. Gieseler, C. Moritz, C. Dellago, R. Quidant, and L. Novotny, Phys. Rev. Lett. 116, 243601 (2016).

[12] A. A. Clerk, M. H. Devoret, S. M. Girvin, F. Marquardt, and R. J. Schoelkopf, Rev. Mod. Phys. 82, 1155 (2010).

[13] H. van de Hulst, Light Scattering by Small Particles, Dover Books on Physics (Dover, New York, 1981).

[14] F. Tebbenjohanns, M. Frimmer, and L. Novotny, Phys. Rev. A 100, 043821 (2019).

[15] V. Salakhutdinov, M. Sondermann, L. Carbone, E. Giacobino, A. Bramati, and G. Leuchs, Optica 3, 1181 (2016).

[16] V. Salakhutdinov, doctoral thesis, Friedrich-AlexanderUniversität Erlangen-Nürnberg (FAU), 2020, urn:nbn:de:bvb: 29-opus4-137383.

[17] A. K. Swan, L. A. Moiseev, C. R. Cantor, B. Davis, S. B. Ippolito, W. C. Karl, B. B. Goldberg, and M. S. Unlu, IEEE J. Sel. Top. Quantum Electron. 9, 294 (2003).

[18] B. J. Davis, M. Dogan, B. B. Goldberg, W. C. Karl, M. S. Ünlü, and A. K. Swan, J. Opt. Soc. Am. A 24, 3762 (2007).

[19] P. Bushev, D. Rotter, A. Wilson, F. Dubin, C. Becher, J. Eschner, R. Blatt, V. Steixner, P. Rabl, and P. Zoller, Phys. Rev. Lett. 96, 043003 (2006).

[20] P. Bushev, G. Hétet, L. Slodička, D. Rotter, M. A. Wilson, F. Schmidt-Kaler, J. Eschner, and R. Blatt, Phys. Rev. Lett. 110, 133602 (2013).

[21] L. Slodička, G. Hétet, N. Röck, S. Gerber, P. Schindler, M. Kumph, M. Hennrich, and R. Blatt, Phys. Rev. A 85, 043401 (2012).
[22] G. Cerchiari, G. Araneda, L. Podhora, L. Slodička, Y. Colombe, and R. Blatt, Phys. Rev. Lett. 127, 063603 (2021).

[23] U. Dorner and P. Zoller, Phys. Rev. A 66, 023816 (2002).

[24] D. E. Chang, C. A. Regal, S. B. Papp, D. J. Wilson, J. Ye, O. Painter, H. J. Kimble, and P. Zoller, Proc. Natl. Acad. Sci. USA 107, 1005 (2010).

[25] F. Tebbenjohanns, M. L. Mattana, M. Rossi, M. Frimmer, and L. Novotny, Nature (London) 595, 378 (2021).

[26] C. Genes, D. Vitali, P. Tombesi, S. Gigan, and M. Aspelmeyer, Phys. Rev. A 77, 033804 (2008).

[27] F. Gittes and C. F. Schmidt, Opt. Lett. 23, 7 (1998).

[28] C. Dawson and J. Bateman, J. Opt. Soc. Am. B 36, 1565 (2019).

[29] B. E. Kane, Phys. Rev. B 82, 115441 (2010).

[30] R. Reimann, M. Doderer, E. Hebestreit, R. Diehl, M. Frimmer, D. Windey, F. Tebbenjohanns, and L. Novotny, Phys. Rev. Lett. 121, 033602 (2018).

[31] F. Tebbenjohanns, M. Frimmer, A. Militaru, V. Jain, and L. Novotny, Phys. Rev. Lett. 122, 223601 (2019).

[32] L. Dania, D. S. Bykov, M. Knoll, P. Mestres, and T. E. Northup, Phys. Rev. Research 3, 013018 (2021).

[33] D. B. Higginbottom, L. Slodička, G. Araneda, L. Lachman, R. Filip, M. Hennrich, and R. Blatt, New J. Phys. 18, 093038 (2016).

[34] P. Bushev, A. Wilson, J. Eschner, C. Raab, F. Schmidt-Kaler, C. Becher, and R. Blatt, Phys. Rev. Lett. 92, 223602 (2004).

[35] G. Hétet, L. Slodička, A. Glätzle, M. Hennrich, and R. Blatt, Phys. Rev. A 82, 063812 (2010).

[36] D. Rotter, M. Mukherjee, F. Dubin, and R. Blatt, New J. Phys. 10, 043011 (2008).

[37] G. Araneda, S. Walser, Y. Colombe, D. B. Higginbottom, J. Volz, R. Blatt, and A. Rauschenbeutel, Nat. Phys. 15, 17 (2019).

[38] G. Cerchiari, L. Dania, and D. S. Bykov, Self-Homodyne (2021), https://doi.org/10.5281/zenodo.4545692.

[39] P. Laven, Mieplot v.4.6, http://www.philiplaven.com/mieplot. htm. 